Variations sur la lettre, le mètre et la mesure :

Shakespeare

\title{
Le jeu de l'amour et de la règle
}

Jacques Darras

\section{(2) OpenEdition \\ Journals}

Édition électronique

URL : http://journals.openedition.org/shakespeare/971

DOI : 10.4000/shakespeare.971

ISSN : 2271-6424

Éditeur

Société Française Shakespeare

Édition imprimée

Date de publication : 1 novembre 1996

Pagination : 71-80

Référence électronique

Jacques Darras, "Le jeu de l'amour et de la règle », Actes des congrès de la Société française

Shakespeare [En ligne], 14 | 1996, mis en ligne le 01 janvier 2007, consulté le 21 avril 2019. URL : http:// journals.openedition.org/shakespeare/971; DOI : 10.4000/shakespeare.971 


\section{Variations sur la lettre,}

le mètre et la mesure

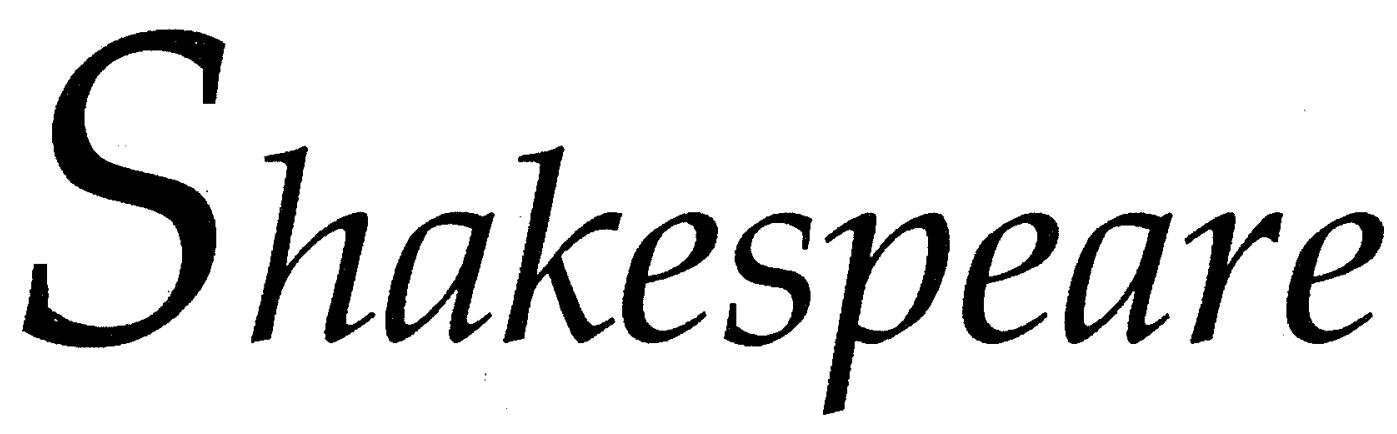

textes présentés par Dominique Goy-Blanquet

Directeur de la publication : Richard Marienstras 


\section{Le jeu de l'amour et de la règle}

Les pires ennemis des poètes ont toujours été les poètes. Dans son introduction au Wheel of Fire de Wilson Knight, Eliot se montre d'une sévérité impitoyable pour l'espèce à laquelle lui-même appartient. La meilleure au demeurant : dans son club personnel il n'y a que trois noms, Lucrèce, Dante et Shakespeare. Mesuré à la balance de la philosophie ce trio ne fait cependant pas le poids, conclut-il, car les poètes ne pensent pas. Certains encore moins que d'autres. Tel Shakespeare, dont on se demande d'ailleurs pourquoi Eliot a accepté de préfacer l'étude le concernant. Soixante ans plus tard, on dirait que ce sentiment est devenu général. La compétition pour l'accès à la vérité privilégie plus que jamais la philosophie. De la considération que les poètes ne pensaient pas, on a très systématiquement induit que la poésie n'avait pas à penser. A plaire tout au plus, à décorer. Les rhétoriques modernes n'infirmant nullement les grammaires classiques sur ce point. Contre Eliot je dirai que non seulement la poésie pense, mais que Shakespeare dramaturge pense prioritairement par la poésie. Que c'est au moment où il est le plus poète dans son discours qu'il formule le plus finement sa pensée. La poésie de Shakespeare est conjugaison d'une pensée et de sa mesure. Commençons par rappeler la citation d'Eliot :

J'ai toujours été d'avis que non seulement Shakespeare n'était pas poète philosophique au sens de Dante et Lucrèce mais encore que les "poètes philosophiques" style Dante et Lucrèce ne sont pas de véritables philosophes.... J'ai affirmé un jour que Dante avait fait de la grande poésie à partir d'une grande philosophie de l'existence alors que Shakespeare avait fait sa non moins grande poésie à partir d'une philosophie de l'existence inférieure et confuse. Je n'ai pas de raison sérieuse de modifier cette affirmation

Entre poètes et philosophes, le point d'affrontement le plus manifeste est la métaphore. Sa qualité d'énigme suscite un incoercible désir d'appropriation par la raison. Excessive, la métaphore donne à entendre que le langage se déborde, se tient en réserve de lui-même. La métaphore pose au fond des opérations linguistiques un principe de liberté totale qui menace l'ordre rationnel du monde. Bizarrement, ce métaphoricien suprême que fut Shakespeare a nourri fort peu de discussions sur le sujet. Critiques et metteurs en scène travaillent toujours sur le schéma des grammaires anciennes, confortés en cela par ce que j'appellerai "l'idéalisme historiciste" contemporain. "Idéalisme", car prétendre reconstituer le cadre d'un écrivain passé revient à restreindre à son horizon phénoménologique d'alors la 
virtualité de désordre toujours contenue dans son langage. Cela brime évidemment l'horizon de ceux qui viennent ensuite comme Wilson Knight défendre les fondements d'une critique d'interprétation. Prenons Mesure pour mesure. Le titre est éloquent, et l'action correspond au titre. Dans cette construction exemplairement adroite, reposant sur effets de symétrie, de surprise, de renversement, l'esprit de comédie se risque comme l'équilibriste sur le fil tendu et coupant de la tragédie. Vincentio encourt manifestement plus de risques dans son duché de Vienne que Prospero sur son île antillaise. Mais pour nuancée qu'elle paraisse, la leçon de gouvernement politique est finalement simple. Appel généralisé à la tempérance, la tolérance, l'humanisation des lois. La pièce vaut d'un bout à l'autre par son ironie dramatique. Nous, spectateurs, sommes rendus complices par le sourire, à la fois notre paiement et notre rétribution - measure for measure. Faut-il pour autant conclure avec T. S Eliot à une modicité, une mendicité philosophique de circonstance?

Il en va de Shakespeare comme de La Fontaine. Chez eux le dénouement est souvent moins passionnant que le "nouement". Non pas ce que les Italiens appellent "l'imbroglio" - l'embrouillamini. Plutôt l'entrelacement réciproque du dramatique et du rhétorique, poésie et langage. La métaphore ici n'est pas fil brillant, passementerie ornementale qui suivrait la grammaire classique des figures et des tropes - comme on dit "filer la métaphore" - mais torsion ensemble de l'action et des mots, d'où naît la pensée. La pensée comme tension. La poésie comme action. Exemple. Quand Isabella vient plaider auprès d'Angelo la cause de son frère Claudio condamné à mort, nous assistons à un cours de persuasion poétique. Tel un conseiller en communication, Lucio invite la jeune fille à échauffer son discours, à passer la "vitesse poétique" supérieure pour mieux émouvoir l'intraitable tyran. Comme toujours dans la comédie shakespearienne, l'instrument et son fonctionnement sont exposés en même temps. Le discours d'Isabelle se transforme devant nous à mesure de sa plaidoirie, l'image poétique prend, tend sa parole jusqu'à ce que, comble d'ironie ! les critiques de Lucio glissent peu à peu au contrepoint d'harmonie. La poésie, plaide Shakespeare, agit, la poésie "marche", parce qu'elle fait accéder l'action à un palier supérieur où l'équation dramatique change de signe. Circonvenu dans la rafale d'images qu'Isabella fait éclater sur sa tête, Angelo baisse pavillon. Il n'a de toute évidence pas assez de conviction au fond de lui pour prendre la mesure poétique du discours adverse. La densité de la foudre métaphorique l'accable - tout comme elle éblouit le spectateur. Sa teneur est telle qu'on soupçonnerait presque une manœuvre rhétorique de diversion. La vulgarité des encouragements de Lucio transformant le débat en un match de boxe n'est pas faite pour nous rassurer. Mais l'effort surhumain auquel se hausse le discours d'Isabella ne cherche pas tant l'obscurité que l'inspiration d'une vérité supérieure. Au ciel, qui est la plus haute source à laquelle s'adresse la métaphore, Isabella cueille la condensation lumineuse de l'éclair. Or cette "condensation" de lumière a pour effet paradoxal d'opérer une division. L'éclair métaphorique tranche. La poésie chez Shakespeare peut agir comme une arme, un glaive de justice sur l'adversaire "prosaïque" : 


\section{Could great men thunder}

As Jove himself does, Jove would ne'er be quiet

For every pelting petty officer

Would use his heaven for thunder; nothing but thunder.

Merciful heaven,

Thou rather with thy sharp and sulphurous bolt

Splits the unwedgeable and gnarled oak,

Than the soft myrtle. But man proud man,

Dress'd in a little brief authority,

Most ignorant of what he's most assured-

His glassy essence - like an angry ape

Plays such fantastic tricks before high heaven

As makes the angels weep; who with our spleens,

Would all themselves laugh mortal.

Maniée par Isabella la poésie devient l'arme, l'armure maillée de son innocence. L'inextricable réseau d'images où s'entrecroisent arbres de la forêt et arbres de la foudre - où éditeurs et traducteurs s'aventurent souvent comme dans un taillis, la serpe à la main - signifie que la logique métaphorique ne se pénètre pas. Ne se résume pas. Ne se déplie pas. Demeure rebelle à l'explication qui voudrait la maîtriser. La métaphore crée un sanctuaire intérieur au langage qui laisse tout juste entrevoir l'idée obliquement - brillante et obscure. Etre poète c'est chaque fois faire appel à l'inviolable du divin dont la parole ne peut se faire entendre que par procuration. Ainsi, courant au-devant d'elle-même dans son balbutiement néophyte, Isabella lève une profusion d'images qui semblent se contredire. L'important est qu'elle crée un espace de tension supérieur aux lois de la cité. La poésie tient en effet sa force d'une conjonction des contraires qu'aucune dialectique "civile" ne peut raisonnablement admettre. Platon, son désir d'ordre "républicain" sont ici de nouveau défiés. La poésie divise, la poésie désorganise. Isabella partie, Angelo reste à débattre (se débattre) au milieu d'un impossible "waste land" :

Having waste ground enough

Shall we desire to raze the sanctuary

And pitch our evils there?

"Terre vaine" contre "sanctuaire", Isabella a ouvert une question qui n'est pas près de se refermer. On ne se "réfugie" pas sans risque à l'improviste dans le "sanctuaire" de la poésie. Il n'y règne pas la loi civile. Il n'y règne que du désir et de la séduction. Angelo l'a compris. Le recours à l'arme poétique fait pénétrer Isabella à l'envers de la loi, dans une économie de prostitution généralisée où les corps sont échangeables. Non pas par principe d'équivalence absolue mais accroissement métaphorique de leurs qualités respectives - ce que disent ailleurs à leur manière les Sonnets. Un monde de métaphores est un monde d'amour total, de corruption totale. Les Titanias y folâtrent avec les ânes. Franchi le seuil, la comédie devient métaphore généralisée. Pour faire bonne "mesure", on substitue ainsi successi- 
vement le corps de Mariana à celui d'Isabella, la tête du pirate Ragozine à celle de Claudio : une valse viennoise des figures qui ramènera finalement l'ordre civil sur les rives du Danube. On ne peut pas vivre constamment dans la substitution. Il faut un régulateur des échanges poétiques sous peine que l'enchantement devienne anarchie. Ici le maître des échanges Vincentio se trouve très heureusement détenir aussi le pouvoir politique. Le drame met fin à la poésie.

Sauf lorsqu'il y a obstination à vivre poétiquement jusqu'au bout. C'est le cas de quelques personnages. Au premier rang Richard II, que la "métaphore royale" prend pour ainsi dire en otage et qui finira sa vie en "poésie" comme d'autres "en réclusion". L'entêtement de ce Plantagenêt né en Aquitaine, formé à la courtoisie aspect souvent négligé - atteint à une véritable magie cérémoniale. Dans son cas la poésie rejoint progressivement la religion, et ce roi le Roi Christ. Quant au coup̄le formé par Antoine et Cléopâtre, il ne devrait pas moins susciter la curiosité. Manifestement cette pièce présente des difficultés de représentation liées à sa structure poétique. En 1947, dans ce qui demeure à ce jour la meilleure étude d'ensemble sur Shakespeare, Henri Fluchère distinguait deux rhétoriques dans son théâtre, l'une dramatique l'autre poétique. Pour la première fois les images faisaient l'objet d'autre chose que d'une plate étude thématique. Fluchère reconnaissait à la métaphore shakespearienne un pouvoir de "violence faite à l'abstraction, qui donne forme poétique à l'allégorie et qui, par accumulation des images, tend à épuiser la substance même de l'objet à décrire et à saisir". Violence à l'abstraction, c'est indéniable. Par contre sur la qualité d' "épuisement" il faut apporter des nuances, des correctifs. Ainsi l'Isabella de Mesure pour mesure cherche-t-elle son salut dans la métaphore comme on se jette à l'eau, manifestant une volonté "dramatique" de combler un manque de crédibilité plutôt que d'épuiser "la substance de l'objet". Pour la sœur de Claudio il s'agit de prendre le temps de vitesse, de porter la contradiction au cœur même d'Angelo par passage à la "raison poétique supérieure". Ici la métaphore a fonction dramatique. Il est souvent difficile pour les critiques de s'affranchir des conceptions classiques de l'image. "Aucune règle rigoureuse, concède lui-même Fluchère, ne nous permet d'affirmer à quel moment nous passons du plan de la rhétorique au plan psychologique et même au plan métaphysique, dans l'utilisation de l'image". Là est en effet la question. L'insistance des grammaires classiques à rapporter les figures à leur "valeur d'usage" apporte des contraintes insoutenables. Il est faux, voire absurde de parler d' "utilisation" métaphysique de la métaphore. Reconnaissons plutôt qu'un métaphoricien comme Shakespeare exprime une métaphysique au cœur même de sa poésie. C'est moins à la notion d'épuisement qu'il faut réfléchir ici qu'à la notion de "vacance". La "poésie" chez Shakespeare semble en effet ne donner toute sa mesure qu'en l' "absence" - par inefficacité, vacance de pouvoir, enchantement — de la règle politique. Lorsque le pouvoir revient les images s'en vont. Ainsi Hamlet "poète" exhale-t-il et sublime-t-il sa souffrance dans les brèves "vacances" de l'action dramatique. On pourrait dire qu'à chaque tournant, chaque nouvelle scène, Shakespeare "pèse" la part qui revient respectivement au dramatique et au poétique. Là est l'objet constant de son attention. 
Une vacance règne au cœur d'Antoine et Cléopâtre. Voire un air de "grandes vacances". L'Egypte des Ptolémées n'est certes pas encore un Club Méditerranée mais la puissance coloniale romaine - plus tard britannique - a déjà œuvré dans cette direction. Enobarbus décrivant sa vision du Nil aux cadres supérieurs de l'Empire est, à la manière de Lucio, un merveilleux publicitaire d'agence de voyages. Toutefois la désinvolture, autrement dit le désengagement "cavalier" qu'on sent chez les uns et les autres, correspond manifestement à une étape audacieuse de la réflexion shakespearienne. Mieux que ne le feront en leur temps Graham Greene ou E. M. Forster, Shakespeare nous livre d'abord son sentiment sur l'esprit colonial. Savoir que l'engluement dans le plaisir dénature l'austérité républicaine de la conquête. Car, suggère-t-il finement, l'intention impériale emporte toujours dans ses bagages une volonté originelle de plaisir. D'où les altérations, les corruptions à venir. Que Shakespeare ne condamne pas. Ce n'est pas. son propos. Ce qui l'intéresse est ailleurs. Ce qui l'intéresse ce sont les conditions de possibilité du bonheur "poétique". Peut-on vivre en couple, sur la terre, en poésie, demande-t-il. Peut-on concentrer la "mesure poétique" de telle manière qu'on interrompe, qu'on suspende le cours, la course prosaïque du drame. Aucune autre pièce n'est plus difficile à représenter, pourquoi ? Parce que les actes, les actions n'y sont pas tant velléitaires que nécessairement morcelés. L'espace est distendu non seulement par la géographie mais par le déplacement rapide de l'action : la division à l'infini des scènes tente de compenser l'extension du drame aux confins de l'univers. D'où un vide central, une "vacance" que le triumvirat des politiques est incapable de combler - the centre does not hold, le centre ne tient plus, dira Yeats en période "édouardienne". Le reflux du politique appelle l'afflux du poétique. Quelle plus merveilleuse image que celle d'Antoine complètement lâché par la foule accourue aux rives du Nil pour voir passer la barque de Cléopâtre et à qui il ne reste plus qu'à siffler, puérilement, dans la "vacance" de l'air autour de lui :

\author{
and Antony, \\ Enthron'd $i^{\prime}$ the market-place, did sit alone, \\ Whistling to the air; which, but for vacancy, \\ Had gone to gaze on Cleopatra too, \\ And made a gap in nature.
}

Comme toujours une foule d'images, d'informations, qu'Enobarbus déploie pour enchanter son auditoire. Or capture et captation sont précisément au centre du récit de Shakespeare. Que se passe-t-il, réfléchit le poète, lorsque délaissant leur propre centre de gravité - politique, domestique - des hommes, des femmes, entrent dans la captivité réciproque du désir ? Qu'en est-il lorsque l'infini, l'instable, l'insatiable désir poétique se fiche au cœur du monde ? S'il est avéré que la chasse d'amour se termine logiquement par une mise à mort, Shakespeare lui-même chasseur qui s'enchante de la course de ses proies s'approche ici dangereusement d'une rupture de cohérence. La pièce devient pour ainsi dire menacée par la "vacance" poétique qui étend son sanctuaire au désert numidien. Antoine et Cléopâtre ont délibérément choisi la métaphore, la légende. Non seulement eux la 
reconnaissent, mais cela est dit dans l'écriture exceptionnellement condensée, concentrée, elliptique du vers. Désertant le nœud dramatique de son canevas, Shakespeare tente l'impossible. Se risquer aux limites de sa découverte : son art lui a appris qu'il y a un vide infranchissable entre langage de légende et langage d'action. Qu'il y a donc soupçon sur la cohérence de l'univers divin. Que l'utopie, par définition impossible, ne peut se concevoir que comme hyperbole (est-ce la vision hérö̈que du christianisme ?) dans un continuel évitement du centre. Qu'il n'y a, poussant la réflexion plus loin, pas de réconciliation possible - sinon conventionnelle comme au théâtre - entre le Dieu de la Loi et le Dieu de l'Amour.

L'enjeu d'Antoine et Cléopâtre dépasse de loin les problèmes politiques, il touche à la métaphysique de la division. Triumvirat contre couple, trois puis un contre deux, quelle est la bonne arithmétique ? Un auteur aussi généreusement scindé en plusieurs morceaux de lui-même - tel le serpent - est inévi-tablement conduit à s'interroger sur le principe de division. Et s'il est poète, sur la place de la métaphore, qui brouille tout. La métaphore est manifestement deux. Un de trop pour Dieu, un de moins que le trois de la Trinité. Que faire du "couple métaphorique" qui s'obstine à d'inconvenantes copulations ? Aristote que la biologie n'intimidait pourtant pas y voyait une figure de la "substitution". La métaphore, ayons enfin le courage de l'admettre, est enlacement cru, accou-plement revendiqué comme tel par la poésie amoureuse. Antoine et Cléopâtre déborde de métaphores. On dirait d'une crue nilienne, d'un orgasme amoureux qui n'en finirait pas. Comme on n'en finit plus de compter ou dénombrer les images de ce transport - cette métaphore égyptienne prolongée. "Nay, but this dotage of our general's/ O'erflows the measure" : dès le premier vers de la première scène, par la bouche du bien nommé Philo, l'ami d'Antoine, le ton est donné. Les incessantes métaphores qui suivent ne débordent pas seulement comme le Nil. Elles s'ordonnent en table, en échelle de chiffres et d'évaluations appliquée au moindre fait amoureux et guerrier. L'amour, la poésie expriment une obsession "comparative". Cléopâtre: "If it be love tell me how much". Partout la démesure porte la marque d'une mesure sur son envers. Disons par plaisanterie que les centimètres de taille manquant à Cléopâtre lui font hausser son langage. Mais surtout Shakespeare confond ici dans une synthèse impériale l'empire romain et l'empire élisabéthain naissant pour les soumettre utopiquement au joug poétique de l'hyperbole. Célébration posthume de la geste courtoise, sursaut de la philosophie médiévale pour introduire la graduation poétique dans la plate réalité, c'est ainsi que sonne à nos oreilles l'éloge final d'Antoine par Cléopâtre. Plus tard viendra l'heure des matérialistes, des littéraux, des exégètes, des juristes - en somme des Romains :

\section{For his bounty,}

There was no winter in't : an autumn 't was

That grew the more by reaping : his delights

Were dolphin-like, they show'd his back above

The element they lived in : in his livery

Walk'd crowns and crownets : realms and islands were

As plates dropp'd fromp his pocket. 
Mille ans plus tard Venise est devenue l'Alexandrie de l'Occident. La poésie y rencontre un test plus décisif que celui du pouvoir politique. Celui de l'argent. On n'a jamais bien élucidé la fascination réciproque de ces deux médiateurs invisibles. C'est pourtant le sujet profond du Marchand de Venise. Débat des deux Testaments, l'Ancien le Nouveau, préfère-t-on généralement invoquer. N'est-ce pas faire trop bon marché de la "part poétique" au profit de la "part dramatique" ? Gardons-nous de dévaluer la pensée subtile, indécise de Shakespeare en la rabattant machinalement vers les grandes références philosophiques. Sauver Shakespeare contre Eliot, oui, mais pas à n'importe quel prix. Il faut avancer dans le raisonnement en tenant ensemble métaphore et pensée de la métaphore. Nulle part, dans aucune autre comédie, la pointe sur laquelle tient le drame n'est plus aiguë. C'est une pointe de couteau. Lame merveilleusement polie, affûtée par Shakespeare pour y faire tenir en équilibre simultané comédie et tragédie. A tout moment le "fléau" pourra basculer dans l'un ou l'autre sens. C'est une ingéniosité instrumentale simple qui représente en vérité un sommet d'abstraction théâtrale. Thêâtre, c'est peser les destins. A chaque instant, tout y est renversement, virtualité de renversement. Les hommes, les personnages en équilibre au suspens d'une bascule ressortissent d'un tribunal des "référés" dont les jugements peuvent être bêtement mécaniques ou subtilement réfléchis. En choisissant la pointe d'un couteau qui peut aussi bien se rétracter que faire saigner la chair d'Antonio, Shakespeare dépasse le simple mécanisme de l'oscillation. Ce qu'il tente dans le Marchand de Venise c'est plus abstraitement qu'ailleurs de peser la balance elle-même. De mesurer la mesure.

Dans ce théâtre philosophique voici pourtant que le plus abstrait s'avère être le plus concret. La machine à peser la totalité du destin, âme corps compris, prétend ne prélever qu'une symbolique, partielle "livre de chair". Cette parcellisation met tout le monde en émoi. Confier quatre cents grammes de sa chair aux mains de la chirurgie vénitienne représente d'ailleurs à l'époque un risque non négligeable, Commencini le montrera encore dans son Enfance de Casanova. Le trouble majeur n'est cependant pas là. L'enjeu tient dans l'indécision de la décision. Ou de l'incision. L'opération est trop grave pour prêter à rire, mais pas suffisamment pour que le drame bascule dans la tragédie. Il faut voir ici un suspens de même nature que le "doute suspensif" de Descartes. Accrochant le drame à la pointe fine métaphorique de l'expression "livre de chair", Shakespare prélève sa part d'attention du spectateur pour la réflexion. Le problème posé est celui du corps de la vérité. Du corps sur quoi est gagée la vérité. D'un côté Shylock, véritable matérialiste avant la lettre, plaide pour une réalité "terrestre" qui n'est pas le tout. Qui ne sera jamais le tout. Nos corps sont prosaïquement divisibles, dit-il. A leur image la réalité est fragmentaire, comptable, dénombrable, puisque humblement gagée sur cette "dette" inépuisable, sans fond, absolue - au sens de non déliable, non rachetable - qu'est la mort. Il y a donc une limite à la loi des substitutions. Tout n'est pas substituable à tout comme voudrait héroïquement le faire croire la poésie. Une partie seulement l'est - par exemple "livre de chair" pour "dette". Nous vivons dans la métonymie. Jamais dans la métaphore. La partie pour le tout. Nul escamotage, nulle substitution ou déguisement de cette vérité n'est finalement convaincant. La métaphore qui fait si bon marché des corps qu'elle substitue - la rédemption du Christ étant la 
métaphore par excellence, la métaphore royale - ouvre en vérité un risque de dévaluation généralisée. C'est peu de dire que Shylock ne croit pas à l'incarnation. Qu'il ne croit pas à cette substitution d' "amour" introduite par le Christianisme. Qu'il ne croit ni à la métaphore ni à la métamorphose "transubstantiatrice". Il démontre cruellement aux Chrétiens que leur religion du "tout incarné" s'affole devant la moindre "parcellisation" douloureuse pour le corps.

Mieux que Hamlet, le Marchand de Venise est la pièce philosophique de Shakespeare. Dont le sujet est la réalité. Le statut de la réalité. Tout poète doit tôt ou tard avoir le courage d'affronter l'ennemi intime qu'il porte en lui - son double, son "autre", son Juif. Doit faire comparaître la comparaison au tribunal de la pensée. Nul ne dèvrait se croire exempt d'un tel examen. Quelle plus belle métaphore que l'expression "livre de chair" ? Pourtant dans le Venise de Shylock quelle réalité plus littérale ? L'habileté chirurgicale de Portia déguisée en avocat - substitut d'avocat - parviendra sans doute à une conciliation. Mais au-delà de l'esprit de comédie qui distribue les récompenses ou les rétributions finales, la question "ouverte" par incision, la plaie, ne se referme pas. Comment le même matériau d'existence peut-il se prêter à deux lectures simultanées contradictoires, l'une minimale l'autre maximale, l'une fragmentaire l'autre absolue, correspondant aux axes croisés de l'économie d'échange monétaire (le prix de la dette) ou d'échange poétique (la substitution des corps dans l'image) ? Irrémédiablement conduit hors la philosophie chrétienne, Shakespeare vit cette expulsion, ce dénudement - au sens où l'expérimentent Richard II et Antoine et Cléopâtre - moins comme exil que comme expérience métaphysique majeure. Il y a une ontologie poétique de Shakespeare qui ne fait pas de l'alternative "être" ou "ne pas être" la question ultime. Hamlet est au mieux un bon élève de Sénèque ou Cicéron par l'enseignement de la Renaissance. Sa réflexion superficielle ne touche pas à l'objet fondamental du drame shakespearien qui est la "réversibilité" de l'être.

Notre auteur réputé non philosophe accomplit de manière originale l'analyse qui occupe depuis Platon la pensée occidentale, distinguer ce qui relève du concept de ce qui relève de l'image. Faut-il laisser la philosophie en faire l'enjeu de sa propre légitimité ? La question est aujourd'hui plus complexe, plus aiguë que jamais. S'appuyer sur Paul Riccur pour dire que la métaphore est "vive" ne suffira pas forcément à délivrer Shakespeare du mépris d'Eliot. Rompant avec les dévoiements "poético-conceptuels" d'un Derrida, Ricœur entend en effet redonner à la méțaphore une place plus conforme à sa noblesse. Qu'est-il prêt à concéder à la poésie ? En apparence beaucoup. "En elle-même et par elle-même, dit-il, la poésie donne à penser l'esquisse d'une conception tensionnelle de la vérité..." Mais "donner à penser", remarquons cependant, n'est pas la même chose qu'être sujet actif de sa propre pensée. Car, poursuit Ricœur, l'expérience poétique révèle notre "appartenance qui inclut l'homme dañs le discours et le discours dans l'être". De sorte, conclut-il, que la poésie "préfigure la distanciation que la pensée spéculative porte à son plus haut degré de réflexion". Autrement dit la poésie ne servirait qu'à fournir les "figures", la matière brute des "semblances et resssemblances", la philosophie se chargeant quant à elle de creuser la réflexion sur leur être ou non 
être, donc sur notre identité. Autrement dit le poète - en l'occurrence Hamlet tendrait en permanence au philosophe le miroir de l'image. Servilement.

Dieu merci la poésie a toujours su allègrement bousculer les rôles où prétendait la confiner la philosophie, alors qu'une étrange alliance semble rapprocher théâtre et philosophie, pareillement travaillés par la même obsession du dénouement. Comme si pour l'un et l'autre il convenait au plus haut point de ne jamais abandonner la maîtrise des régulations. Toute autre est l'économie de Shakespeare. Chez lui la machine métaphorique produit des dérèglements de sens qui ne sont pas des crues de Nil ou de Tamise, des accidents de la superfluité. La métaphore chez Shakespeare est fondamentale. La métaphore est l'essence même de notre "appartenance" au monde. Peut-être relève-t-elle de notre vieille adhésion païenne à l'univers mais plus subtilement, plus douloureusement, la métaphore nous. dit, nous joue le "jeu de la substance" dans quoi nous sommes "incarnés". La métaphore montre à la fois l'opération d'enveloppement par quoi nous rabattons les choses les unes sur les autres, les mesurons l'une à l'autre, et la manière dont nous sommes mesurés, enveloppés, par cette opération poétique même. Etre poète c'est toujours manifester la conscience aiguë de cette double nature - le mesureur mesuré pris dans un double pli de l'être. D'où cette constante présence de la métaphore de l'étoffe, de l'habit (stuff) propre à l'économie marchande élisabéthaine, qui n'a pas seulement habille le discours mais fournit la matière initiale du patron. L'image du coup de ciseaux qui nous taille tous dans le même tissu au début de Mesure pour Mesure est elle aussi retournée retournable. Le dénommé Lucio la retaille comme endroit et doublure : "Well there went but a pair of shears between us/ I grant : as there may between the lists and the velvet". Cependant que maniée par l'adresse de l'avocate-couturière, Portia, la même paire de ciseaux prouve a contrario l'impossibilité de trancher entre figuré et littéral. Tranchez dans le figuré, vous faites saigner le littéral, ou vice et versa. Shakespeare est un métaphysicien de la réversibilité qui habille d'autant mieux sa pensée que la métaphore la déguise.

Jacques DARRAS Université de Picardie 